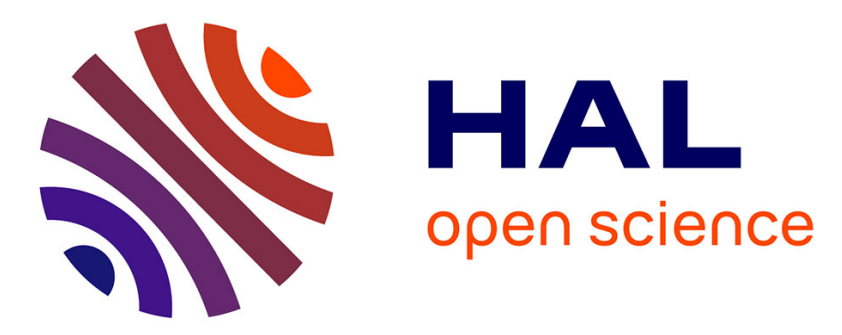

\title{
Influence of constraints on the dynamics of polypeptide chains.
}

Konrad Hinsen, Gérald Kneller

\section{To cite this version:}

Konrad Hinsen, Gérald Kneller. Influence of constraints on the dynamics of polypeptide chains.. Physical Review E , 1995, 52 (6), pp.6868-6874. 10.1103/PhysRevE.52.6868 . hal-02155715

\section{HAL Id: hal-02155715 \\ https://hal.science/hal-02155715}

Submitted on 13 Jun 2019

HAL is a multi-disciplinary open access archive for the deposit and dissemination of scientific research documents, whether they are published or not. The documents may come from teaching and research institutions in France or abroad, or from public or private research centers.
L'archive ouverte pluridisciplinaire HAL, est destinée au dépôt et à la diffusion de documents scientifiques de niveau recherche, publiés ou non, émanant des établissements d'enseignement et de recherche français ou étrangers, des laboratoires publics ou privés. 


\title{
Influence of constraints on the dynamics of polypeptide chains
}

\author{
K. Hinsen* and G.R. Kneller ${ }^{\dagger}$ \\ Institut für Theoretische Physik A, Rheinisch-Westfälische Technische Hochschule Aachen, Templergraben 55, \\ D-52056 Aachen, Germany \\ (Received 16 March 1995; revised manuscript received 26 June 1995)
}

\begin{abstract}
Based on the equations of motion for linked rigid bodies that we derived recently [G. Kneller and K. Hinsen, Phys Rev. E 50, 1559 (1994)], we develop a technique for the simulation of molecular systems with constraints. We apply it to analyze the importance of the various degrees of freedom of a polypeptide chain for its dynamics. We find that keeping the peptide planes rigid does not change the dynamics much, but that the bending degrees of freedom of the $\alpha$-carbon bond geometry are essential for large-amplitude backbone motions. This means that the $\phi$ and $\psi$ angles commonly used to characterize protein conformations and protein backbone dynamics do not constitute a sufficient set of variables to perform dynamical simulations.
\end{abstract}

PACS number(s): $02.70 . \mathrm{Ns}, 87.15 . \mathrm{He}$

\section{INTRODUCTION}

One of the major goals in the development of simulation techniques is the reduction of the computer time needed to run simulations of complex systems. The use of constraints to eliminate fast motions and thereby allow larger time steps in molecular-dynamics (MD) simulations is already a standard technique [2]. Besides, the use of rigid subunits in macromolecules holds the promise of allowing a simplification of the force fields [3]. Since the calculation of the forces accounts for most of the CPU time in MD simulations, this approach deserves serious consideration.

The most commonly used method to implement constraints is known as SHAKE [4]. SHAKE uses the same set of Cartesian coordinates that describes the atomic positions of the corresponding unconstrained system. A list of bond constraints can be imposed by computing for each time step constraint forces from a system of quadratic equations describing the constraints. This set of quadratic equations must be solved iteratively. For strongly interdependent constraints the convergence can be very poor. In some cases it is impossible to calculate constraint forces from the list of bond constraints: linear rigid molecules with more than two atoms and planar rigid molecules with more than three atoms. In these cases and in the case of three-dimensional rigid molecules with more than four atoms, one may also have redundant constraints, which yield an incorrect number of degrees of freedom. An improved method to handle such systems

\footnotetext{
${ }^{*}$ Present address: Département de Chimie, Université de Montréal, C.P. 6128, Succursale A, Montréal, Québec, Canada H3C 3J7. Electronic address:

hinsenk@ere.umontreal.ca

${ }^{\dagger}$ Electronic address: g.kneller@kfa-juelich.de
}

has been developed [5], but it is not in general use. In this method the constraints are split into bond constraints for 2,3 , or 4 "basis atoms," and linear constraints, which relate the positions of the remaining atoms to those of the basis atoms. Chains of topologically linked rigid units cannot be treated at all with the iterative methods developed so far [6]. Examples for such systems are doublering structures in which the two rings have a common axis, e.g., the protein side-chain thyrosin, and the socalled "calixarenes," which basically consist of a closed chain of benzene rings.

One possibility to avoid the problems of iterative methods is the use of generalized coordinates that correspond to the remaining degrees of freedom of the constrained system. This is a common approach in theoretical classical mechanics [7], but it is rarely used for numerical calculations. The commonly quoted reason is that finding the right set of coordinates and deriving the equations of motion for them is too complicated and has to be redone for each new system or set of constraints to be studied. Some of the attempts in this direction $[9,10]$ seem to confirm this view. However, a realistic simulation of $n$-butane using generalized coordinates has been published in [11], but it should be noted that the equations of motion derived in that paper are incorrect in that they do not take into account the normalization conditions for quaternions.

In a recent paper [1], we have derived equations of motion for topologically linked rigid bodies, which represent a very general class of geometrical constraints. We have also shown how a set of generalized coordinates can be constructed for such systems. Our approach covers all atomic systems with distance constraints, excluding for the moment closed chains, which will be treated later. In this paper, we will show how a general and easy-to-use simulation algorithm can be constructed on the basis of this theoretical treatment. Taking a list of constrained distances as input, this algorithm will automatically find the appropriate generalized coordinates and integrate the 
corresponding equations of motion. It makes it possible to simulate complicated constrained systems without manual intervention.

As an application, we analyze the influence of various sets of constraints on the dynamics of a polypeptide chain. An earlier study [8] of the effect of freezing all bonds and all bond angles on the dynamics of a protein concluded that while it is safe to freeze covalent bonds, freezing all bond angles leads to significant changes in dynamical quantities. In this work, we compare two more physically motivated sets of constraints: rigid peptide planes, and a model that leaves only the $\phi$ and $\psi$ angles (i.e., rotations around the peptide bonds) unconstrained, thus corresponding to the set of variables commonly used to describe protein backbone conformations. Simulations in these coordinates have been used successfully in crystallographic structure refinement [12]. We will show, however, that they do not constitute a sufficient set of variables to perform dynamical simulations of helices, whereas keeping the peptide planes rigid does not lead to significant changes.

\section{IMPLEMENTATION}

\section{A. Equations of motion}

In [1] we have derived equations of motion for flexible chains made of rigid subunits, each consisting of two or more atoms. This derivation is based on the following consistent choice of generalized coordinates and velocities: (i) The orientation of each rigid subunit is described by four components of a normalized quaternion. (ii) The rotation of each rigid subunit is described by a set of angular velocity components. (iii) The position and translational velocity of the whole chain in space is described by the position and velocity of an arbitrary reference atom. In analogy to the treatment of conventional rigid-body dynamics [7], we use angular velocities as generalized velocities for rotational motion instead of the time derivatives of the angular coordinates. As we have described in [1], the matrix $C_{i k}(i=1, \ldots, 3 N ; k=1, \ldots, f)$, which maps the generalized velocities $u_{k}$ onto the Cartesian velocities $\dot{r}_{i}$ via

$$
\dot{r}_{i}=C_{i k}\left(x_{\alpha}\right) u_{k}
$$

then has a simple structure. It depends on the positions of all atoms and reflects the connectivity of the chain as well as the types of joints between the subunits. Here and in the following, we assume summation over pairwise like indices. The chain is supposed to consist of $N$ atoms and have $f$ degrees of freedom. Its configuration is described by the generalized coordinates $x_{\alpha}\left(\alpha=1, \ldots, n_{c}\right)$, which contain the three Cartesian coordinates of an arbitrary reference atom and one normalized quaternion for each rigid subunit. Due to the use of quaternions, $n_{c}$ is larger than $f$, but the coordinates $x_{\alpha}$ are subject to $n_{c}-f$ normalization conditions. The generalized velocities $u_{l}(l=1, \ldots, f)$ comprise the three Cartesian veloc- ities for the reference atom and a set of angular velocity components for each rigid subunit.

Writing the Lagrangian of the system in the coordinates described above and taking into account the normalization conditions for the quaternion parameters, we derived the following equations of motion:

$$
\begin{aligned}
M_{i j} C_{i k}\left(x_{\alpha}\right) \frac{d}{d t}\left[C_{j l}\left(x_{\beta}\right) u_{l}\right] & =F_{m} C_{m k}\left(x_{\gamma}\right), \\
\dot{x}_{\alpha} & =B_{\alpha i}\left(x_{\beta}\right) u_{i} .
\end{aligned}
$$

The matrix $M_{i j}$ is diagonal and contains the masses of all atoms. The matrix $B_{\alpha i}\left(\alpha=1, \ldots, n_{c} ; i=1, \ldots, f\right)$ relates the generalized velocities to the time derivatives of the generalized coordinates. Due to the use of quaternions these relations can be written in a singularity-free form. The construction of the matrices $B_{\alpha i}$ is described in detail in [1]. In the following we will show how these equations of motion can be used to design an integrator for an arbitrary atomic system with distance constraints that avoids many of the problems arising with iterative methods such as SHAKE $[4,5]$.

\section{B. Topological analysis}

A crucial step in the implementation of our method is the calculation of the matrix $C$ defined by (1), which gives the relation between the generalized velocities of the flexible chain and the Cartesian velocities of its constituent atoms. This matrix depends on the current position of all atoms (which are easily available) and on the constraints being imposed. As has been shown in Sec. III B of [1], the matrix $C$ contains information about which atoms are part of which rigid subunit(s), as well as about the way in which these subunits are linked to each other. Obtaining this information manually for a nontrivial system is a formidable and error-prone task. It is better to leave this work to the computer.

We have implemented an automatic topology analyzer that takes a list of constrained distances as input and finds all rigid subunits in the system, as well as the connections between them and the degrees of freedom for each subunit. The presence of redundant distance constraints in the list poses no problems, which it does for algorithms that satisfy all constraints iteratively [4]. Although the restriction to distance constraints may seem a limitation, almost all types of constraints that are typically encountered in the simulation of molecular systems can easily be expressed in terms of a list of distance constraints. An even larger class of constraints can be handled with the addition of massless dummy atoms to the system. An example of a constrained system that cannot be described in this way is a linear molecule whose bond lengths are not fixed (i.e., there are only angle constraints, but no bond-length constraints). It is possible to extend the topology analyzer for such systems if the need arises.

The input to the topology analyzer is the list of distance constraints and the starting configuration of the system; the latter is needed only to detect linear con- 


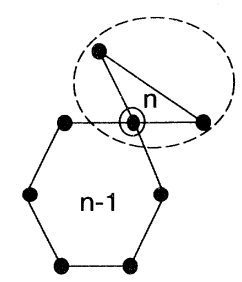

(a) 3 degrees of freedom

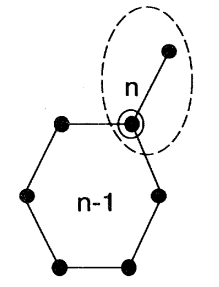

(b) 2 degrees of freedom

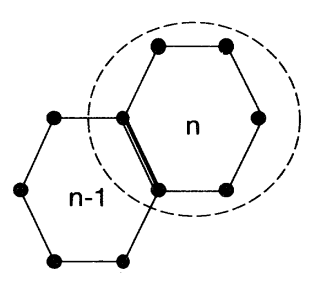

(c) 1 degree of freedom
FIG. 1. Illustration of the different types of joints describing the links between succeeding rigid bodies $n$ and $n+1$. From left to right: (a) the joint allows for free rotation, (b) the joint allows for free rotation, but due to the linear structure of unit $n+1$ its angular velocity must not have a component along its axis, (c) the angular velocity of unit $n+1$ must be parallel to the common axis of unit $n+1$ and $n$. figurations of more than two atoms. The output of the topology analyzer is a list of flexible chains and a list of unconstrained atoms. Each flexible chain is described by a list of rigid subunits. For each rigid subunit, the analyzer determines the type of motion relative to the subunit to which it is attached, and the atom(s) it has in common with that subunit. There are four possible types of motion (see Fig. 1): (i) Translation of one reference point in every chain. (ii) Rotation with three degrees of freedom. This means that the subunit has one atom in common with the subunit to which it is attached. (iii) Rotation with two degrees of freedom. This occurs for rigid subunits that are linear and therefore have no degree of freedom corresponding to rotation around their own axis. (iv) Rotation with one degree of freedom. This means that the subunit has an axis (two or more atoms along a straight line) in common with the subunit to which it is attached. The only degree of freedom is rotation around this axis.

\section{Finding the rigid subunits}

The first step in the topological analysis is the determination of rigid subunits, which are constructed atom by atom from the list of constraints. The algorithm is based on two rules: (i) Three atoms whose three pair distances are constrained form a rigid unit. (ii) An atom whose distance from at least three atoms of a rigid unit is constrained is itself part of the same rigid unit. In practice these simple rules are complicated by additional considerations for groups of more than two atoms located on a line, and by the bookkeeping needed to treat atoms that are part of more than one rigid subunit correctly. In the following exact description of the algorithm, the word "linked" refers to two atoms whose distance is fixed, either because a distance constraint was given in the input list, or because both atoms are part of the same rigid subunit being constructed: (1) Find a group of three atoms linked to each other. At least one of the three atoms must not already be part of a rigid subunit. These three atoms form a new rigid subunit. If no such group can be found, continue with step (4). (2) Find an atom linked to three atoms of the rigid subunit under construction. The three atoms must not lie on a straight line. Add the new atom to the subunit, and add the distances between it and all previous atoms of the subunit to the list of constrained distances. Repeat this step until no further atom to be added can be found. (3) Continue with step (1). (4) Find all constrained distances that are not inside rigid subunits. Each of them refers to a new rigid subunit consisting of two atoms.

\section{Finding chains of rigid subunits}

The second step consists of constructing the flexible chains out of the rigid subunits. This is done by looking for atoms that belong to more than one subunit. Two subunits can either have one atom or one axis (two or more atoms along a line) in common. If they had more common atoms, they would in fact be only one rigid subunit and would have been identified as such in the preceding step. (1) Generate a list of all atoms that are common to two or more rigid subunits. (2) Pick an arbitrary rigid subunit that is not already part of a chain and make it the first member of a new chain. Pick an arbitrary atom in this subunit and assign three translational degrees of freedom to it. Assign three rotational degrees of freedom for rotation of this subunit around the chosen reference point. (If the subunit is linear, assign only two degrees of freedom.) If no more rigid subunits are available, terminate. (3) In the list generated in step (1), find an atom that is contained in a rigid subunit that is already part of a chain. Add the other subunit(s) that contain it to the same chain. Assign rotational degrees of freedom to each of these subunits according to their linearity and the number of atoms they share with their predecessor. Repeat this step until no more rigid subunits to be added can be found. (4) Continue with step (2).

At the end of this algorithm, all the necessary data for the calculation of the matrix $C$ is available. It should be noted that this analysis needs to be done only once for a system in which the constraints do not change in time. It is also worth mentioning that the topological analysis described above can also be used to implement iterative methods designed for rigid units of more than three atoms [5], although no such method has yet been developed for chains containing more than one rigid subunit with three or more atoms.

\section{Integration of the equations of motion}

The topological analysis described in the previous section provides a list of flexible chains and a list of all atoms 
that are not part of any chain. The latter require no special treatment and their trajectories are obtained with a standard leap-frog integrator. For each of the chains we have to integrate the equations of motion (2) and (3). Equation (2) can be written as

$$
\dot{u}_{l}=\left(\Theta^{-1}\right)_{l k} C_{m k}\left(x_{\gamma}\right)\left[F_{m}-M_{m n} \dot{C}_{n p}\left(x_{\beta}\right) u_{p}\right]
$$

with

$$
\Theta_{k l}=M_{i j} C_{i k}\left(x_{\alpha}\right) C_{j l}\left(x_{\beta}\right)
$$

The last term on the right-hand side of (4) is quadratic in the velocities; it is similar to the centrifugal and Coriolis forces occurring in rotating reference frames. The presence of this velocity-dependent force necessitates modifications to the standard integration schemes used in molecular-dynamics applications [11], which also results in a better estimation of the kinetic energy [13].

To facilitate the description of our integration algorithm, we rewrite Eqs. (4) and (3) in the form

$$
\begin{aligned}
& \dot{\mathbf{u}}=[\boldsymbol{\Theta}(\mathbf{x})]^{-1}[\mathbf{k}(\mathbf{x})+\mathbf{g}(\mathbf{x}, \mathbf{u})] \\
& \dot{\mathbf{x}}=\mathbf{B}(\mathbf{x}) \mathbf{u}
\end{aligned}
$$

where $\mathbf{k}(\mathbf{x}) \quad=[\mathbf{C}(\mathbf{x})]^{T} \mathbf{f}(\mathbf{x})$ and $\mathbf{g}(\mathbf{x}, \mathbf{u})$ $=[\mathbf{C}(\mathbf{x})]^{T} \mathbf{M} \dot{\mathbf{C}}(\mathbf{x}, \mathbf{u}) \mathbf{u}$. We will write $\mathbf{x}_{n}, \mathbf{u}_{n}$ to denote the values of $\mathbf{x}$ and $\mathbf{u}$ at the discrete time step $n$. Our integrator is based on a leap-frog scheme and will therefore at each iteration calculate $\mathbf{u}_{n+1 / 2}$ and $\mathbf{x}_{n+1}$ using earlier values of $\mathbf{x}$ and $\mathbf{u}$ and the forces $\mathbf{f}\left(\mathbf{x}_{n}\right)$. The algorithm for one time step $\Delta t$ is as follows: (1) Calculate $\Theta\left(\mathbf{x}_{n}\right)$. (2) Solve

$$
\boldsymbol{\Theta}\left(\mathbf{x}_{n}\right)\left(\mathbf{u}_{n+1 / 2}-\mathbf{u}_{n-1 / 2}\right)=\Delta t\left[\mathbf{k}\left(\mathbf{x}_{n}\right)+\mathbf{g}\left(\mathbf{x}_{n}, \mathbf{u}_{n}\right)\right]
$$

iteratively for $\mathbf{u}_{n+1 / 2}$, estimating $\mathbf{u}_{n}$ by

$$
\mathbf{u}_{n} \approx \frac{3}{8} \mathbf{u}_{n+1 / 2}+\frac{6}{8} \mathbf{u}_{n-1 / 2}-\frac{1}{8} \mathbf{u}_{n-3 / 2} .
$$

For the first iteration, use $\mathbf{u}_{n} \approx \mathbf{u}_{n-1 / 2}$. (3) Solve

$$
\mathbf{x}_{n+1}-\mathbf{x}_{n}=\Delta t \mathbf{B}\left(\mathbf{x}_{n+1 / 2}\right) \mathbf{u}_{n+1 / 2}
$$

for $\mathbf{x}_{n+1}$, estimating $\mathbf{x}_{n+1 / 2}$ by

$$
\mathbf{x}_{n+1 / 2} \approx \frac{3}{8} \mathbf{x}_{n+1}+\frac{6}{8} \mathbf{x}_{n}-\frac{1}{8} \mathbf{x}_{n-1} .
$$

(4) Normalize the quaternions in $\mathbf{x}_{n+1}$.

We now make some remarks on the individual steps. (1) For reasons described in the following section, we use the center of mass of each chain as the reference point for translation. (2) The estimate given in Eq. (8) is more accurate than the simpler estimate $\mathbf{u}_{n} \approx$ $\left(\mathbf{u}_{n-1 / 2}+\mathbf{u}_{n+1 / 2}\right) / 2$; it is also used for the calculation of the kinetic energy. The repeated solution of the linear system of equations with the coefficient matrix $\Theta$ is done by calculating the Cholesky factorization of $\Theta$ once after step (1) and repeating only the back substitution during the iteration. (3) In contrast to Eq. (7), Eq. (9) is linear in the unknown $\left(\mathbf{x}_{n+1 / 2}\right)$ and can therefore be solved analytically. Again Eq. (10) is more accurate than $\mathbf{x}_{n+1 / 2} \approx\left(\mathbf{x}_{n}+\mathbf{x}_{n+1}\right) / 2$. (4) The equation of motion (3) guarantees that $\mathbf{x}$ is normalized at all times. The explicit normalization is made necessary by the discretization error in Eq. (9).

\section{Center-of-mass coordinates}

In [1] we have stated that any atom in a chain can be chosen as the reference point for the global translational motion. This is true as far as the equations derived in [1] are concerned, but not all choices are equally good for the numerical stability of an implementation. If the reference atom is chosen badly, e.g., close to one end of a long and almost linear chain, the elements of the inertia matrix $\Theta$ in Eq. (5) will range in magnitude from the mass of a single atom to the mass of the whole chain; i.e., the inertia matrix will be ill conditioned. The best reference point from a numerical point of view would be the center of mass of the chain. This would have the added advantage that the translational motion would be uncoupled from the rotational degrees of freedom.

However, it is not possible to construct the constraint matrix $\mathbf{C}$ as described in [1] with the center of mass as a reference point, since the location of the center of mass depends on all coordinates of the chain. It is, however, possible to construct the constraint matrix using an arbitrary atom as the reference point and then calculate the center-of-mass version from it. First we rewrite Eq. (1) as

$$
\dot{r}_{i}^{(n)}=\dot{r}_{i}^{(R)}+C_{(n) i k}^{\prime}\left(x_{\alpha}\right) u_{k}^{\prime}
$$

Here the index $R$ refers to the reference atom, the index $i$ runs from 1 to 3 , the index $n$ runs from 1 to $N, u_{k}^{\prime}$ contains only the rotational velocities (therefore $k$ runs from 1 to $f-3)$, and $C_{(n) i k}^{\prime}\left(x_{\alpha}\right)$ contains only the elements of $\mathbf{C}$ referring to rotational degrees of freedom. What we want is a relation of the form

$$
\dot{r}_{i}^{(n)}=\dot{r}_{i}^{(\mathrm{c} . \mathrm{m} .)}+C_{(n) i k}^{(\mathrm{c} . \mathrm{m} .)}\left(x_{\alpha}\right) u_{k}^{\prime}
$$

Since the center-of-mass velocity is given by

$$
\dot{r}_{i}^{\text {(c.m.) }}=\frac{1}{M} \sum_{n=1}^{N} m_{n} \dot{r}_{i}^{(n)}
$$

where $m_{n}$ is the mass of atom $n$ and $M=\sum_{n} m_{n}$ is the mass of the whole chain, $C_{(n) i k}^{(\text {c.m. })}$ must be given by

$$
C_{(n) i k}^{(\mathrm{c.m.})}\left(x_{\alpha}\right)=C_{(n) i k}^{\prime}\left(x_{\alpha}\right)-\frac{1}{M} \sum_{n=1}^{N} m_{n} C_{(n) i k}^{\prime}\left(x_{\alpha}\right)
$$

which follows from inserting Eq. (11) into Eq. (13), in-

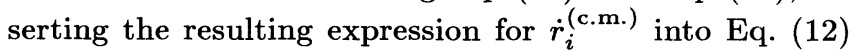
and comparing to Eq. (11).

\section{POLYPEPTIDE CHAINS}

As an example for a polypeptide chain we selected a polyalanine molecule in vacuum made up of 16 peptide 
groups and consisting of 99 "extended atoms" (i.e., $\mathrm{CH}_{n}$ groups are treated as single atoms, so that only polar hydrogen atoms are represented explicitly). We have chosen this example because polyalanine folds into a so-called $\alpha$-helix, which is a common structural element in proteins. Changes in the dynamics of such a regular structure due to geometrical constraints can be easily detected and characterized. Moreover, since the $\phi$ and $\psi$ angles are the standard variables to characterize backbone conformations, it is a natural question to ask whether these variables would also be useful for the description of backbone dynamics.

We studied three models differing in their degrees of freedom: (1) Only the bonds are frozen. Bond vibrations are known to be decoupled from the other dynamical degrees of freedom [8]. In our case, this leaves 199 degrees of freedom. (2) All bonds are frozen, and the peptide planes are kept rigid. There are 150 degrees of freedom in this model. (3) All bonds and all bond angles are frozen, and the peptide planes are kept rigid. This leaves only the $\phi$ and $\psi$ angles unconstrained, i.e., the dihedral angles defining the orientation of the peptide groups (see Fig. 2). This model leaves 38 degrees of freedom for our polyanaline molecule. For each model we performed an MD simulation over a time of $82 \mathrm{ps,} \mathrm{after} \mathrm{an} \mathrm{equilibra-}$ tion run of $10 \mathrm{ps}$. The time step for the integration was $1 \mathrm{fs}$.

The first model (frozen bonds only) was run using the GROMOS package of computer programs [14]. The other two models were simulated using the analyzer-integrator described above and the GROMOs force field.

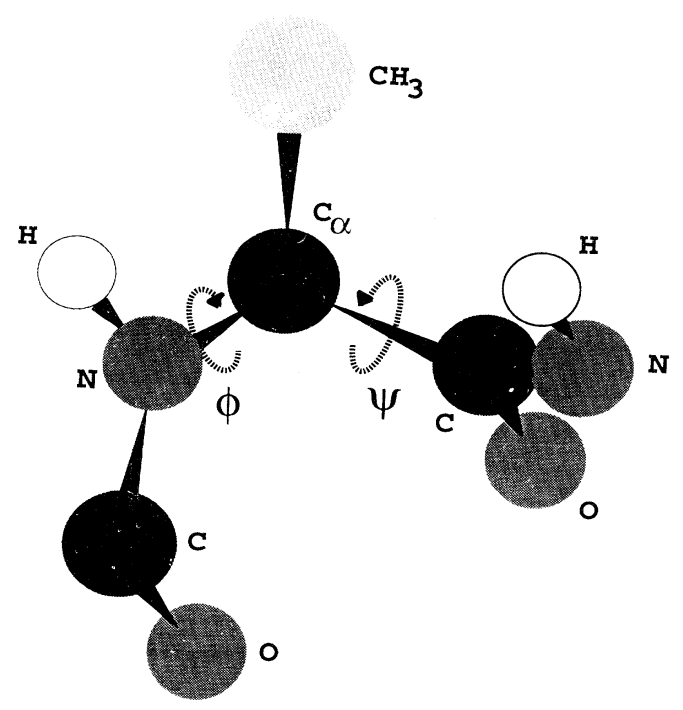

FIG. 2. A section of the polypeptide chain, showing two peptide groups and the $C_{\alpha}$ atom linking them. The dihedral angles $\phi$ and $\psi$ are indicated.

\section{A. Results}

In Fig. 3 we show configurations of the backbone of the polypeptide chain at different time steps superimposed onto each other [15]. The picture clearly shows that the model with 38 degrees of freedom, i.e., with only $\phi$ and $\psi$ angles, is much less flexible than the other two models. This can also be seen in a quantitative way from the mean-square displacement. Figure 4 shows the average over the mean-square displacements of all atoms in the polypeptide chain, i.e.,

$$
W(t)=\frac{1}{N} \sum_{j}\left\langle\left[\mathbf{r}_{j}(t)-\mathbf{r}_{j}(0)\right]^{2}\right\rangle,
$$

where $N$ is the number of atoms in the chain and $\mathbf{r}_{j}$ is the position of extended atom $j$. It is evident that dynamics in $\phi$ and $\psi$ space produces results that are not satisfactory. The two other models, however, lead to qualitatively and quantitatively similar behavior.

To analyze why so much information is lost in the most rigid model, we have calculated the power spectrum of the velocity autocorrelation function, again as an average over all atoms:

$$
P(\omega)=\frac{1}{N} \sum_{j} \int_{0}^{\infty} d t \cos (\omega t) C_{v v}^{(j)}(t)
$$

with

$$
C_{v v}^{(j)}(t)=\left\langle\mathbf{v}_{j}(t) \cdot \mathbf{v}_{j}(0)\right\rangle .
$$

The results are shown in Figs. 5 and 6 . Since the meansquare displacement is related to the power spectrum by

$$
W(t)=\frac{2}{\pi} \int_{-\infty}^{\infty} d \omega \frac{1}{\omega^{2}} P(\omega)(1-\cos \omega t)
$$

it is only the low-frequency part that gives a substantial contribution. Figure 6 shows that all contributions below $40 \mathrm{~cm}^{-1}$ are missing in the model with 38 degrees of
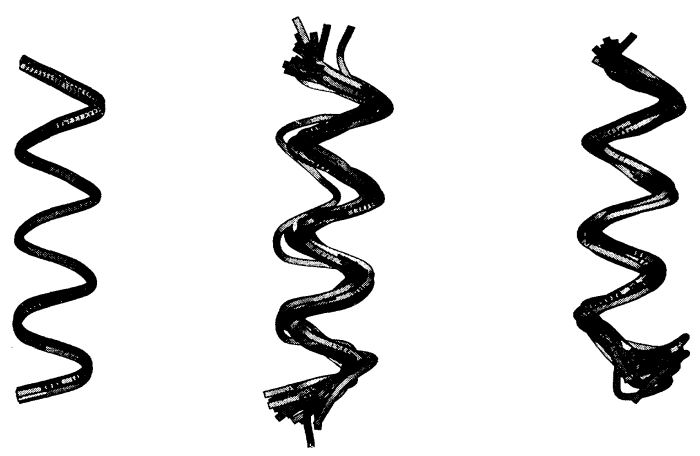

FIG. 3. The backbone of the polypeptide chain at different time steps. From left to right: the models with 38, 150, and 199 degrees of freedom [15]. 


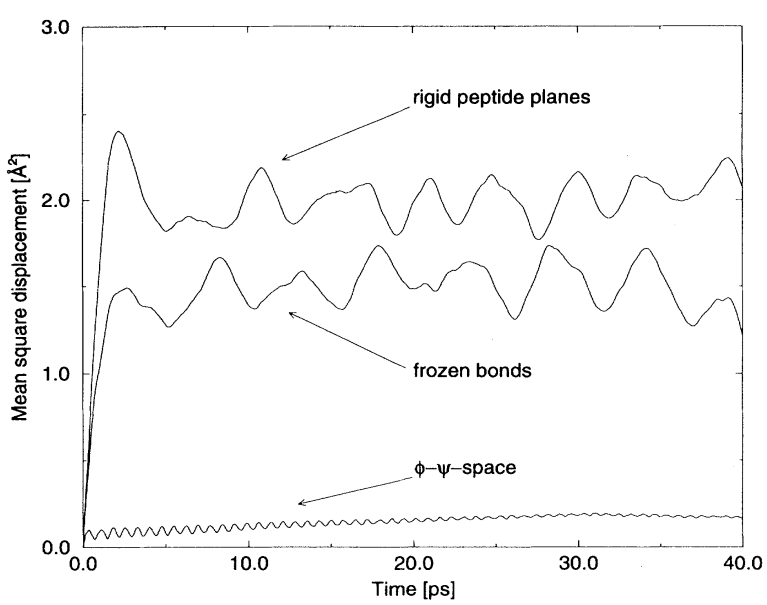

FIG. 4. The average of the atomic mean-square displacements for the three models.

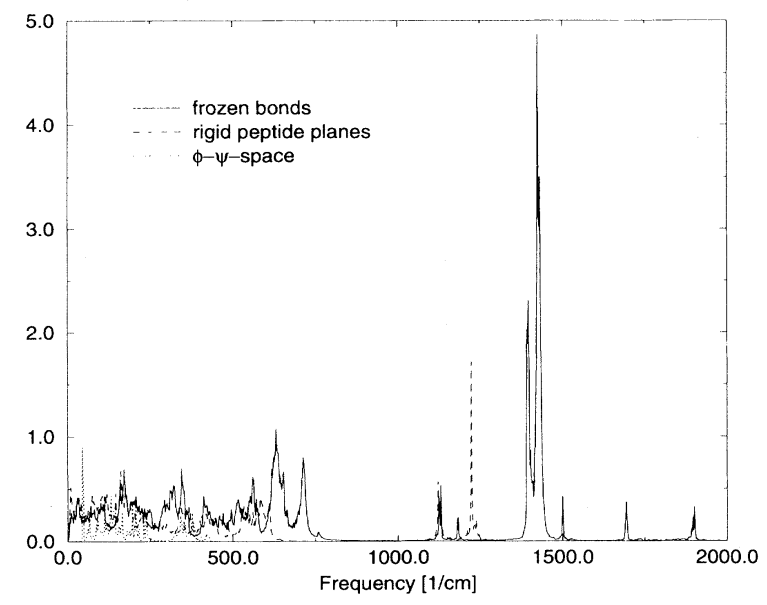

FIG. 5. The power spectrum of the average of the atomic velocity autocorrelation functions for the three models.

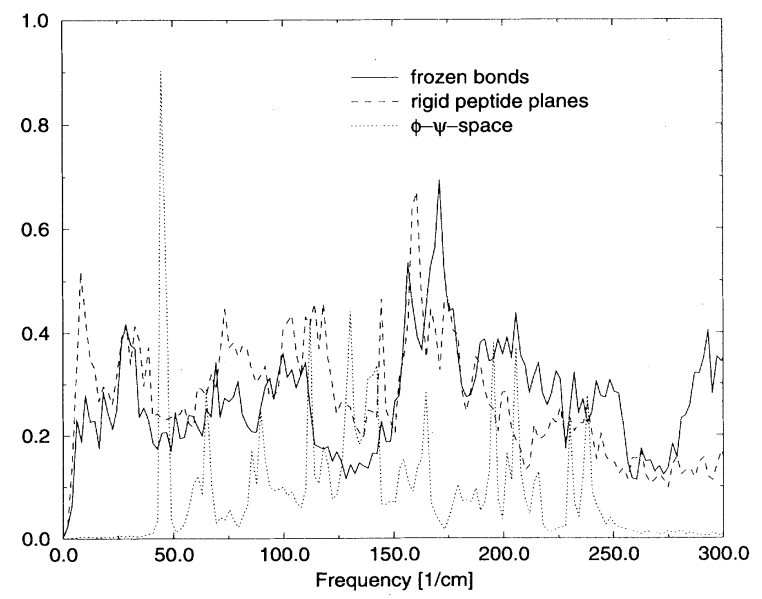

FIG. 6. The low-frequency part of Fig. 5. freedom. The sharp peak at $40 \mathrm{~cm}^{-1}$ corresponds to the fast oscillations that can be seen in the mean-square displacement. The model with rigid peptid planes, however, correctly reproduces the frequencies up to $500 \mathrm{~cm}^{-1}$.

The slight overestimation of the mean-square displacement may be due to a weaker damping of the helix bending motion caused by the rigid peptide planes, which cannot partially absorb this type of motion by internal deformations. This interpretation is confirmed by comparing the low-frequency part of the power spectra $P(\omega)$ for 199 and 150 degrees of freedom (Fig. 6). The strong peak at about $10 \mathrm{~cm}^{-1}$ corresponding to helix bending motion is less pronounced in the flexible model. In this context we emphasize that we do not use a force field that is adapted to our model, but a full atomic force field. A reparametrization of the force field would be necessary to obtain quantitatively correct low-frequency dynamics.

The low-frequency modes that are missing in the $\phi-\psi$ model are those that represent collective motions of large parts of the molecule, e.g., bending modes of the $\alpha$ helix. The only collective motion allowed by the constraints is a torsional vibration. An animated visualization of the molecular-dynamics trajectories clearly shows that the absence of bending modes has a profound influence on the dynamics of a polypeptide chain [16], and this expectation is numerically confirmed by our results for the mean-square displacements and the power spectrum of the velocity autocorrelation function. Nevertheless, it should be noted that this very simple model can still be useful for applications where dynamic quantities are not needed, as in crystallographic structure refinement [12].

\section{CONCLUSION}

We have presented a new method to handle constraints in molecular-dynamics simulations that allows us to simulate systems with arbitrary distance constraints easily and without having to worry about redundant constraints or convergence problems for specific geometries. We have applied this method to study the relevance of various degrees of freedom for the dynamics of a polypeptide chain. We find that keeping the peptide groups rigid does not modify the dynamics significantly, but that a simulation with $\phi$ and $\psi$ angles only shows an important lack of flexibility.

Undoubtedly further work is necessary to establish the essential degrees of freedom of more realistic systems, i.e., larger molecules surrounded by a solvent. We expect our basic conclusion to remain valid: that it is possible to perform realistic simulations while keeping selected groups of atoms rigid. This would allow the construction of simplified force fields for such rigid groups, e.g., using an approach similar to the one presented in [3], which would reduce the computer CPU time spent on the most expensive part of an MD simulation.

\section{ACKNOWLEDGMENTS}

We thank Dr. P. Krüger and G. Kurapkat for help with setting up the polypeptide chain and with using the GROMOS package. 
[1] G. Kneller and K. Hinsen, Phys. Rev. E 50, 1559 (1994).

[2] M.P. Allen and D.J. Tildesley, Computer Simulation of Liquids (Oxford University Press, Oxford, 1987).

[3] T. Head-Gordon and C.L. Brooks III, Biopolymers 31, 77 (1991).

[4] J.P. Ryckaert, G. Ciccotti, and H.J.C. Berendsen, J. Comput. Phys. 23, 327 (1977).

[5] G. Ciccotti, M. Ferrario, and J.-P. Ryckaert, Mol. Phys. 47, 1253 (1982).

[6] J.P. Ryckaert (private communication).

[7] H. Goldstein, Classical Mechanics (Addison-Wesley, Reading, 1971).

[8] W.F. van Gunsteren and M. Karplus, Macromolecules 15, 1528 (1982).

[9] A.K. Mazur and R.A. Abagyan, J. Biomol. Struct. Dyn. 6, 815 (1989).
[10] A.K. Mazur, V.E. Doroveev, and R.A. Abagyan, J. Comput. Phys. 92, 261 (1991).

[11] K. Refson and G.S. Pawley, Mol. Phys. 61, 669 (1987).

[12] L.M. Rice and A.T. Brünger, Proteins 19, 277 (1994).

[13] M. Amini and D. Fincham, Comput. Phys. Commun. 56, 313 (1990).

[14] W.F. van Gunsteren and H.J.C. Berendsen, Molecular Simulation (GROMOS) library manual (Biomos, Groningen, 1987).

[15] The figures were produced with the program MOLSCRIPT, P.J. Kraulis, J. Appl. Cryst. 24, 946 (1991).

[16] A video sequence of 3-min length is available to interested readers. Contact one of the authors or Klaudia Waschbuesch, ZAM, KFA Jülich, D-52425 Jülich, Germany (E-mail: kl.waschbuesch@kfa-juelich.de). 


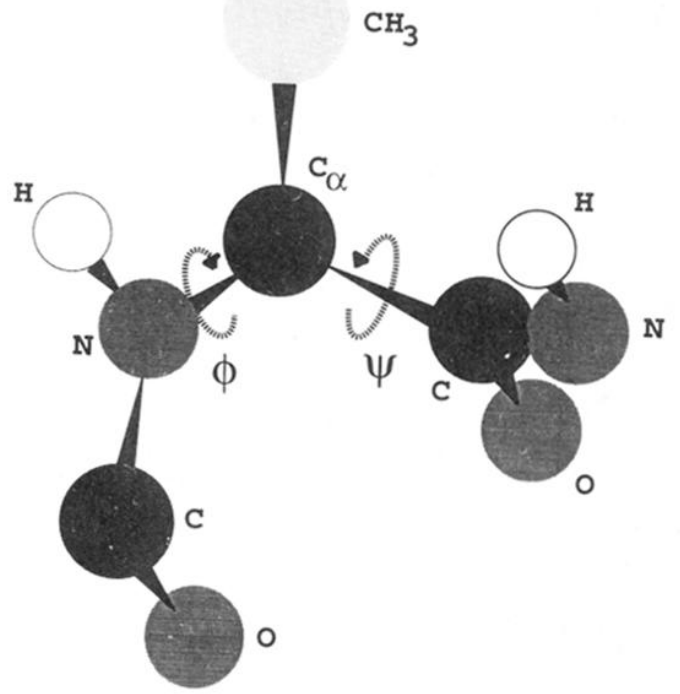

FIG. 2. A section of the polypeptide chain, showing two peptide groups and the $C_{\alpha}$ atom linking them. The dihedral angles $\phi$ and $\psi$ are indicated. 